wo dieses matass noch niclit gegolten hatte? Die bestimmte aufführungszeit konnte darum nicht willkürlich ausgedehnt werden; war also ein stïck unverlı̈̈ltnissmässig länger, so musste dieses an den äbrigen desselben dichters abgeben, und so glaube ich dann auch die anomale erscheinung des Aristias in unserer didaskalie erklären zu können, ohne zu einer auslassung meine zufluclit zu nehmen. Perseus und Tantalos sind tragödien des Aristias selhst, die nicht iiber das gewölnliche matss hinausgehn; mit diesen aber will er ein satyrspiel seines vaters Pratinas verbinden, das noch nach früherer weise auf die liälfte der ganzen aufführung berechnet ist; was hleibt da übrig als die tragüdien bei zweien bewenden zu lassen, vorausgesezt, dass diese mit dem folgenden satyrdrama dieselbe zeit einnelınen, die Aeschylos und Polypliradmon auf ilıre tetralogie verwenden? Und das wäre also ein neuer gewinn an einsiclit in die entwickelungsgeschichte des attischen drama's, wenn meine vermuthung richtig ist, dass ein satyrspiel des Pratinas doppelt so lang zu sein plegte, als es die späteren naclispiele in einer tetelogie waren; gerade darauf aber fülırt schon die angahe des Suidas, dass diese gattung bei Pratinas ebenso stark an zahl als die eigentlichen tragödien war, und zugleich erklärt es sich ganz von selbst, warum bei diesem nocl von keiner tetralogie die rede ist, da ja sogar sein solın, wenn er seines vaters stücke wiederholte, keine solche auf die biihne brachte. Freilich versteht es sich von selhst, diss diese ganze annalıme nur dann zulässig ist, wenn sämmtliclıe stücke des nämlichen dichters hinter einander aufgeführt wurden;

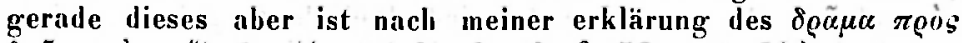

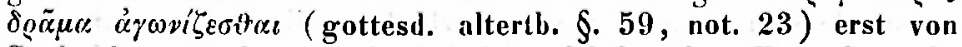
Sophokles geändert worden; und je richtiger lierr Franz bemerkt hat, dass die gewöhnlich angenommene Welckerische erklärung jener worte bereits durcli unsere didaskalie selbst widerlegt wird, desto gesicherter wird, wie icl glaube, die meiuige aucli von dieser seite ersclieinen.

Göttingen.

K. Fr. Hermann.

\title{
30. Parerga.
}

1. Bei Cicero pro Sestio c. 45, §. 97 lıabe ich frülıer (Rh. mus. bd. IV, s. 446) einige handschriftliche lesarten vertheidigt; dagegen kann icli jezt meinen zweifel niclit unterdriicken, oh auch nur richtig lateinisch ausgedrückt sei, was dort in allen handscliriften stelit: sunt maximorum ordinum homines, quibus patet curia. Würden die höchsten stände nicht vielmehr summi ordines lıeissen müssen? und können die näclısten nach dem senate äberall noch maximi lieissen? Ich denke, Cicero sclırieb proxi- 
morum ordinum, wie c. $65, \$ .137$, wo er olınelin zum schlusse seiner episode den gedanken dieses capitels kurz wiederholt. Auch c. 48, \$. 103 nelıme ich anstoss an den worten : nitebantur contra optimates, quod et discordiam excitari videbant et quum locupletes possessionibus diuturnis moverentur, spoliari rem publicam propugnatoribus arbitrabantur. Die ausleger hiaben kein wort darüber gesagt, wie dadurch, dass die begïterten aus langjälırigem besitze vertrieben wurden, der stat seiner vertheidiger beraubt worden sei; denn kriegsdienste konnten jene auch nach geschmälertem grundliesitze immer noch leisten; oder glaubte man, dass ilıre vaterlandsliebe, ihre lingebung für das gemeine wesen durch die erlittene beeinträchtigung heruutergestimmt worden wäre? Wenn mich nicht alles täuscht, so gehört die begründung durch quum - moverentur zu den vorliergehenden worten discordiam excitari videbant; und et muss vor spoliari zu stehen kommen, worauf die zweite befürclitung vielmehr den sinn haben wird, den auch Nitzsch (die Gracchen und ilıre nächsten vorgänger s. 298) nach Appian B. C. I. 7 den gegnern des gracchischen ackergesetzes in den mund gelegt hat: ,ja auch desshalb liegt in der vorgeschlagenen matssregel das verderben der republik, weil man den lifner nicht zum kriegsdienst anziehen oder seine liufe ohne arbeiter lassen darf; entweder wird die republik ohne legrionen, oder das land, das jezt von sclaven wohl bestellt wird, unbebaut sein."

2. Bei Plutarch philos. c. princip. c. 2, p. $777 \mathrm{E}$ schreibt

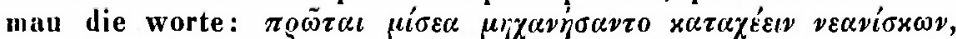
fortwälırend als vers, ohne dass jedoch ein herausgeber so leiclit im stande sein dürfte, maass und rhythmus dieses verses anzugeben. Die sache ist aber höclıst einfach: $\pi \varrho \tilde{\omega} \tau \alpha \iota$ gelı̈̈rt noch zu den vorhergehenden worten Plutarchs, und das übrige bildet einen guten Sotadeus, sobald man nur mit den älteren ausgaben $\mu \dot{v} \sigma \varepsilon \alpha$ liest, was nach Draco p. 65 ein kurzes $v$ hat. Bei demselben ad princ. inerud. c. 6, p. $782 \mathrm{E}$ hat unbegreiflicherweise noch Dübner die verdorbene lesart des bruchstücks aufreclit gelaalten:

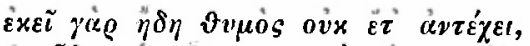

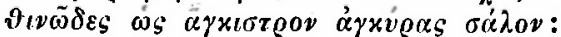

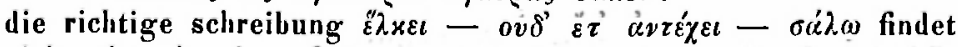
sich schon in einer früheren stelle de virtute morali c. 6, p. $446 \mathrm{~B}$. Schwieriger ist zu verbessern de primo frigido c. 21, p. 955 A :

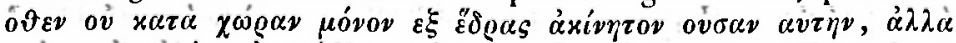

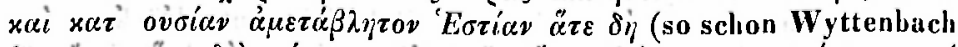

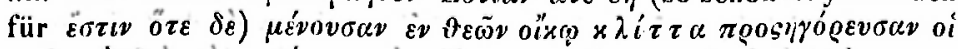

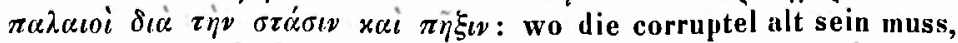
da sich schon in handschriften die sinnlose änderung $\sigma \tau v_{\gamma} \alpha$ findet. Offenbar bedarf es inzwisclien eines wortes für den begriff

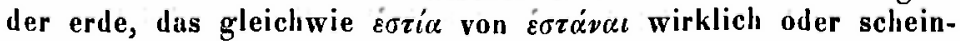


bar von $\pi \eta j v i v a t$ abgeleitet werden konnte, um neben $\dot{\varepsilon} \sigma x^{\prime} \alpha \nu$ deIn folgenden $\pi \tilde{\eta} \xi_{\iota \nu}$ neleen $\sigma \tau \alpha^{\prime} \sigma \nu$ zu entspreclien; und da in $x \lambda_{\iota}$ deutlich xai entlaiten ist, so wird es niclit zu külın seill, in $\tau \tau \alpha$ die ersten züge eines mit $\pi \alpha$ anfangenden wortes zu erblicken, als welches sich am bequemsten $\pi \alpha \gamma a ́ \delta \alpha$ darbietet; vgl. Hesycli.

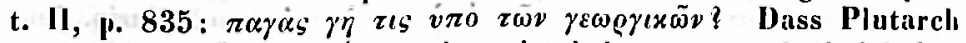

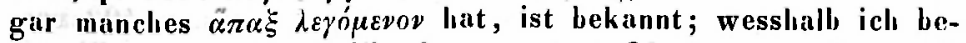
reits im spec. erit. ad lil. de superst. p. 31 zu puer. educ. c. 17 fiir $\lambda v \rho c x i s_{s}$ zu vermuthen gewagt habe $\lambda \alpha \rho \iota x \eta_{s}:$ ein anderes könnte vielleiclıt de cupid. divit. c. 6. der schwierigkeit ablıelfen, dass $x \lambda \eta \emptyset o v \circ \mu о \tilde{u} \sigma \alpha$ unmöglich erbschleichend bedeuten kann; da-

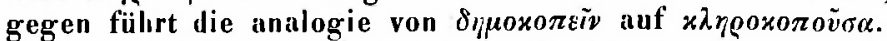

3. Bei Lucrez II, $\mathbf{1 5 0}$ liabe ich früher statt quod sic vermutlıet quod ei diverberat undas; die liandsclıriften aber fülıren auf anderes: Lugd. 1. 2. quo si diverberat, Gottorp. quo si dum diverberat; vielleicht am einfaclisten quasi dum u. s. w. Das, v. 181 :

- nequaquam nobis divinitus esse creatam

naturam mundi, quanquam haec sint praedita culpa;

aber wie passt quanquam zu des dicliters meinung, die aufs deutliclıste eine spätere stelle ausspricht V.199:

nequaquain nobis divinitus esse paratam

naturam rerum; tanta stat praedita culpa?

Die handsclıriften bestätigen ohnehin haec sint nicht; also vielleiclit : quoniam sint oder quoniam st haec, wie man selbst an der andern stelle schreiben könnte: tania st haec. Das. v. 911 :

ab neque aut per se partes sentire necesse est, namque alios sensus membrorum respuit omnis,

widerspricht alios sowohl dem sinne, der jede wahrnelımung der theile ausschliesst, als auch der construction, die dann zu omnis ein besonderes substantiv verlangt; icli lese nam ratio sensus membrorum respuit omneis: der begriff der glieder sträubt siclı gegen alle sinnestluätigkeiten, die ihnen getrennt von ilırem ganzen beigelegt werden könnten. B. IV. 217:

qua re etiam atque etiam mira fateare necesse est corpora, quae feriant oculos visumque lacessant;

olıne sinn, dit der prädicatsinfinitiv zu corpora felitt; vielleicht faleare micare necesse est, d. h. "ِzreı, das auf die durch den

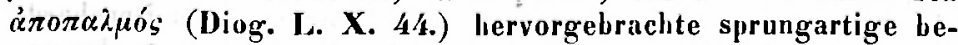
wegung der atome selir gut passt. Das. v. 1185 ist risus doch wolı zu speciell für die mittel, durch welche das weibliclıe geschlecht seinen liebhabern vitae postscenia verbergen will; iclı vermuthe nisus und interpungire ausserdem im folgenden niclit hinter, sondern vor vicissim, so diss dieses zu praelermittere construirt wird.

IV. Aristopl. Plutos v. 352 bietet einen alten aljer docli wie mir sclieint groben feliler dar:

zovzi zovnoov quirezkl zo qogziou. 
Chremylos will dem Blepsidemos sein neues glück mittlieilen, macht aber vor lauter freude umschweife, die diesem verdacht erwecken; was soll da qoeziov? Der sclıoliast erklärt: zo $\beta \alpha^{\prime}$ -

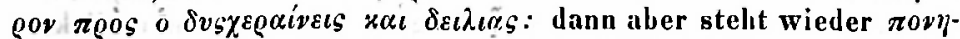
¿òy müssig, und von einer last ist doch überhaupt keine rede;

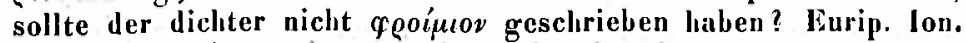

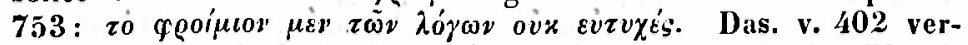
langt cäsur und sinn, dass vò der folgenden rede des Blepsidemos zufalle:

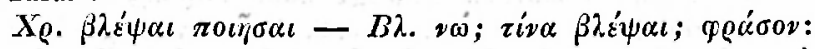
wir zwei sollen jemanden seliend machen? weu denn? sprich! Auclı v. 697 entspriclit die lesirt :

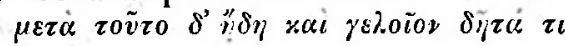

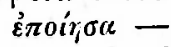

dem vorhergehenden nicht vollig. Die frau fragt: $\delta \delta \varepsilon$ vcòs

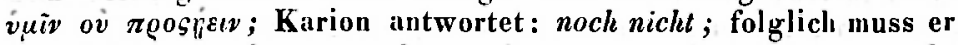
demnächst, wo ein spass, den er in gegenwart des gottes gemacht hat, erzählt wird, vorher erwälınt liaben, dass derselbe inzwischen gekommen sei, was durch änderung von $\eta \delta \eta$ in $\eta \lambda \vartheta \varepsilon$ oder $\ddot{\eta} \varepsilon \iota$ zu bewerkstelligen ist. Und sollte v. 1169 riclitig sein :

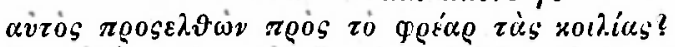

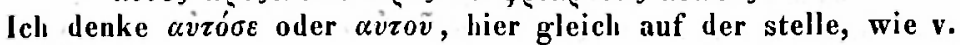
1056 u. s. w.

5. In der erwälınung des gemäldes das Mikon in der $\mathbf{P} \ddot{u}-$ kile bei Zenob. proverb. IV. 28 gefällt mir der berg niclit, auf welchem Butes so gestanden haben soll (' $\varphi^{\prime}$ ' ov $\left.\varepsilon \beta \varepsilon \beta \eta^{\prime} x \varepsilon \iota\right)$, dass man von ilum nur den helm und ein auge salt. Ohnehin scheint es sicher, dass die wandmalereien jener zeit noch ziemlich anilog mit den vasengemälden des attischen styls gelıalten waren (Bullet. dell' Inst. archeol. 1841, p. 4, Welcker Boreas et Oritlıyia p.ı32), und wo bieten diese etwas ähnliches wie einen berg, linter welchem ein mensch liervorsähe? Der fehler ist freilich alt, da ihn schon Suidas nachsclireibt; nichts desto minder aber wage ich fïr ögovs entweder óǵçs oder noch lieber

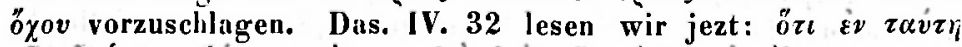

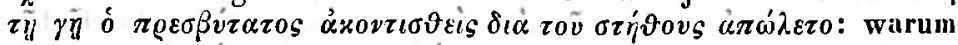

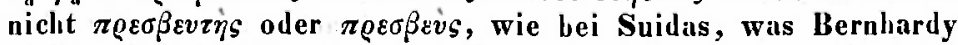
niclit wieder liätte ändern sollen, da das ganze sprichwort:

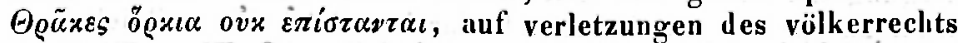

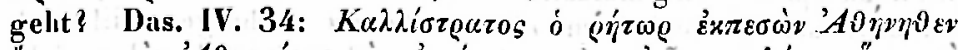

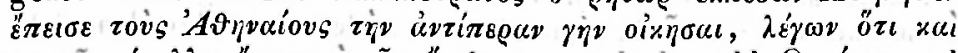

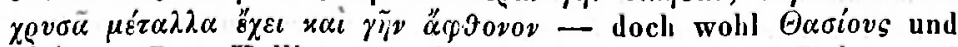

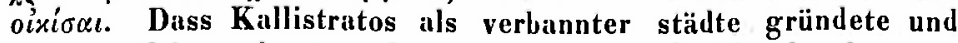
colonien führte, bezeugt Isokrates de Pace $\S$. 24; das konnten aber doch keine athenischen sein; und wirklich lieisst es bei Dio-

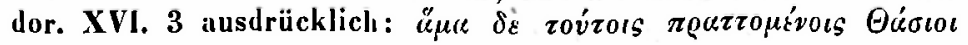




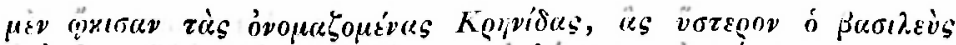

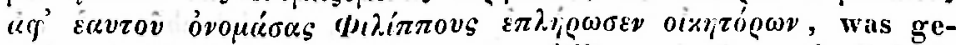
rade mit Kallistratos exil zusammenfällt; vgl. Demostl. Polykl. §. 49.

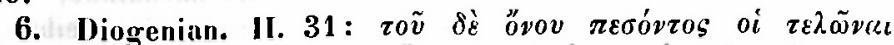

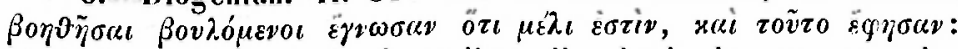

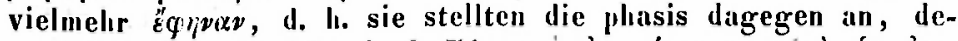

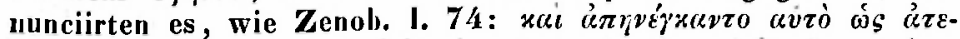

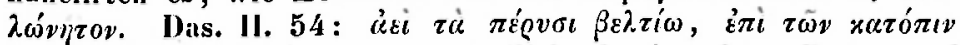

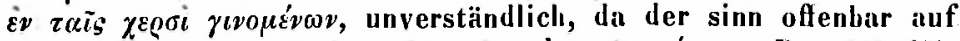

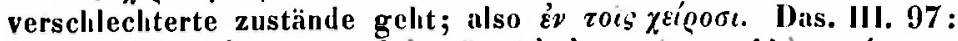

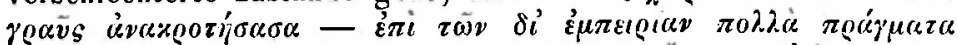

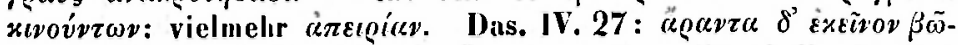

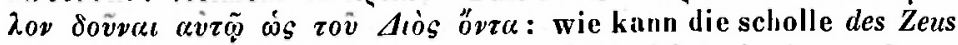
sein? Allerdings ist das spriclıwort frülzeitig mit dem andern : Aios Kó Hesyclı. t. I, p. 1005 und Sclıol. Pindar. Neın. VII. 155; aber ein innerer zusimmenliang ist zwischen beiden um so weniger anzunelımen, je abweichender und scliwankender die meinungen ïber das leztere sind, wälırend das erstere: $\delta \varepsilon^{\prime} \chi \varepsilon \tau \alpha \iota$ $x \alpha \iota \beta \tilde{\omega} \lambda o \nu$ 'Ahíżs lıöclıst einfaclı und klar ist; ja darf man auf den rlıytlımus etwas geben, so rülırt dieses aus einem episclien gedichte, etwa den Kopivoraxois des Eumelos, während jenes in einem solelıen keinen platz findet. Sei also auclı die corruptel noclı so alt, so glaube ich nicht, dass die früleste sage von einem $\beta \tilde{\omega} \lambda_{0}$ s $\tau o \tilde{v}$ Aiós ây etwas wusste, sondern wenigstens in Diogenians quelle is ăg "yrce stand. Aletes bat den lirten um speise, und dieser gab ilın spottend eine sclıolle, als ob es ein brod oder käse wäre, die aber jencr als symbol der besitzergreifung des landes gern annalım; dann erst drehte man die sache so, wie es bei Zenobios 111. 22 beisst, dass der liirt sagt, er liabe kein brod, und statt dessen eine scholle gibt; und endlich wird diese sclıolle, um sie mit Aiós Kógavoos in bezichung zu setzen, selbst zôे Aios, wie bei Hesychios, mag dieses nun anfänglich filsch gelesen oder mit vorbedacht in den text herein interpolirt sein.

7. Bei Athen. VHI. 71, 1. 305 c beginnt das fragment des

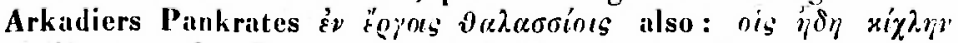
oivw $\delta \varepsilon \varepsilon$ ห. $\pi . \lambda$. Dersatz kïnnte freilich abgebrochen scin, so dass die grammatische beziehung des dativs ois felilte; aber cine solche ist doch schwer zu crgänzen, und viel einfacher ist es, im imperativ zu schreiben : $0 \hat{i} \sigma y_{j} \delta . \tau$. $\lambda$. Das. c. 44, p. 295 a

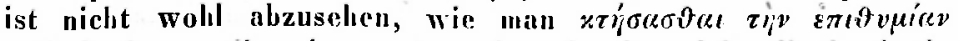
rechtfertigen soll; eine metonymie, durch welche die begierde fiir den begelırten gegrnstand stände, scheint selır hart; also

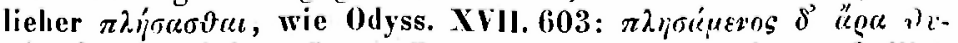

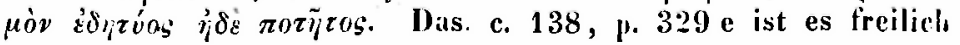

Philulogns, 111 , Jalirg. 3. 
schwer, in einem bruchstïcke, wie das des Antiphanes iv Twe. orrew, genauer über den sinn zu urtheilen; so viel aber ist doch

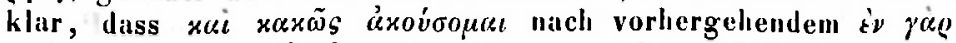

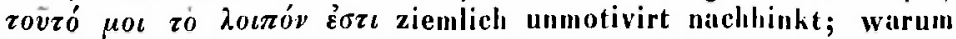
also nicht $x \varepsilon i ?$, "das ist das einzige was mir übrig bleibt, und wenn ich auch darum geschmalit werden sollte."

8. Wie vieles bei Lucian noch zu thun iibrig ist, hat Cobet neuerdings an überraschenden beispielen gezeigt; eine kleine nachlese bietet sich wohl jedem bei erneuerter lesung dar. Prom.

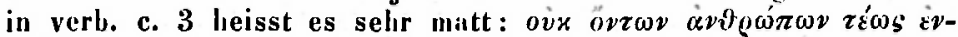

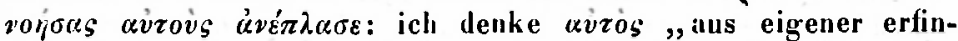

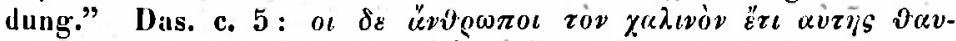

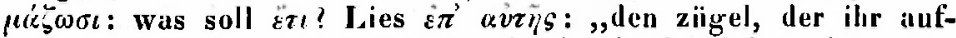

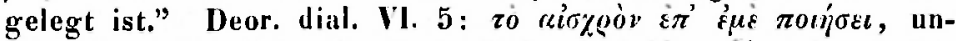
möglich; schon Fritzsche liest mit handschriften $\eta \xi \xi \varepsilon$, das rechte

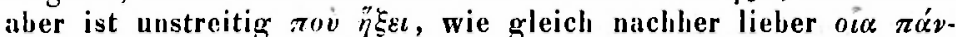

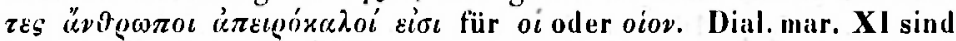

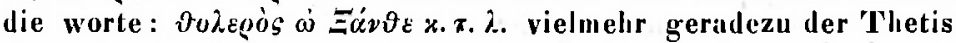
statt der 'Thalatta zu geben, als mit Hemsterhuys viwvov für viov zu schreiben; dass 'Thetis anwesend ist, zeigt schon vorher

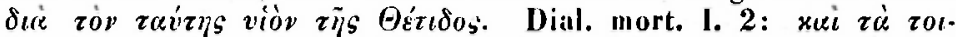

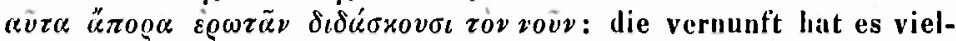
mehr mit ideen als mit syllogismen zu thuu, und die irrlehren der philosophen schaden zunächst der jugend; also zovs véovs.

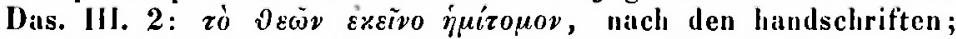
allerdings besser als die vulgatlesart $\vartheta \varepsilon_{0} \tilde{v}$, aber doch nur weil darin noch die spur des richtigen $\vartheta$ gio $\varepsilon \lambda v \pi \eta \sigma \alpha S$ ausser der construction, die vielmelır $\lambda v \pi i j \sigma \alpha S$ fordert.

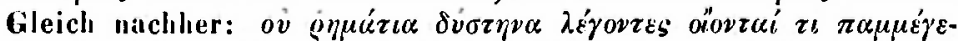

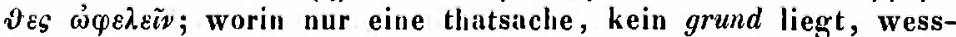
hallb sie vorgezogen werden; diesen gibt ô ônuázıc x. $\tau$. $\lambda$. Das. c. 26 ro $\beta$ iphiov, als ob der philosoplı nur ein buch lä̈tte; ich

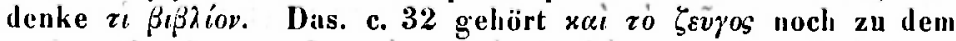

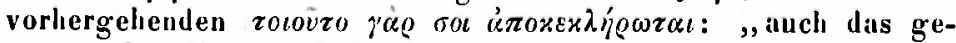
spann, das dir zugetheilt wird, ist so schlecht, dass du zulezt ankommst und dann keine unterkunft melir findest." Das. c. 41:

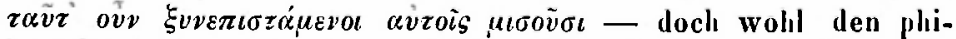
losophen? aber desshalb, weil sie selbst ein böses gewissen haben? ich denke vielmehr, weil er um ilire schlechtigkeit weiss,

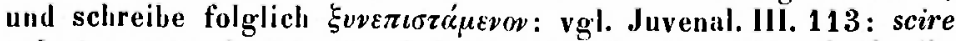
volunt secreta domus atque inde timeri. Scyth. c. 11: o $\delta \dot{\varepsilon}$ vios

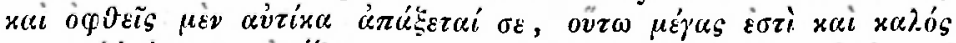

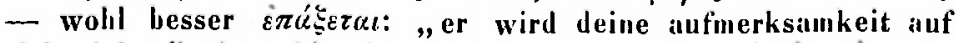
sich ziehen"; das "hinreissen" folgt erst später: $\varepsilon i \quad \delta \varepsilon$ xai po $\vartheta \dot{\gamma}$.

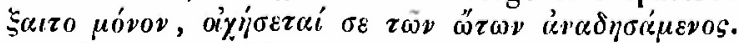

9. Valer. Flacc. Argon. I. 55 wird gewöhnlich so gesclirieben: 
tu cui iam curaeque vigent animique ririles, i decus! et pecoris Nephelaei vellera Graio redde tholo, ac tantis temet dignare periclis.

Hier sclıeint mir vor allem klar, dass decus nicht als anrede an lason gelten darf, sondern zu dem folgenden gehört: $i$ et redde vellera, decus pecoris Nephelaei; ausserdem aber passt mir curae nicht reclit zu vigent uud ich vermisse erwäluung körperliclıer lieldenstärke; also vielleiclıt crura aeque. Auch v. 9' sčlieint mir parentibus die kraft von inexpertos melır zu beschränken als zu steigern, uud dürfte vielleiclit paratibus zu lesen seill. Scliwieriger ist v. 310, wo man obtulit allerdings so deuten kann, dass die morgenrötlıe lason's blicken seine gefährten dargebot $\bullet$ n labe; aber darin liegt doch noch niclıt, was die liauptsache ist und dem folgenden discurrunt transtris den weg bahnen müsste, dass sie sich auf die beine gemacht liaben, und ich möchte dalier lieber abstulit vorschlagen: ,riss sie fort" d. I. braclite sie in thätigkeit. Das. v. 707 visu lassatur inani; ich vermutlıe nisu, du der blick doch einerseits wirklich au dem fluclıtigen Dädalus eineu gegenstand lat, und andererseits das ermüdende wesentlich in dem vergebliclien versuche seiner verfolgung liegt. V. 752 clamore supremo; diclıterisclier wäre supino, wie lıei Martial II. 86: quod nec carmine glorior supino, wo sicli dieselbe variante findet, nur dass es dort durch erhaben, hier durch erhoben übersezt werden muss. II. 29, nimmt man mit recht anstoss an Sicanium dedit usque fretum; aber Burmanns tulit gebt zu weit ab; näher liegt vehit. Das. v. 93 scheint cunclantem gressus selbst für einen dichter dieser zeit zu stark; es genügt conantem. Das. III. 39 befält den steuermann Tiphys ein ticfer sclilaf; wie kann das lieissen: illum non ante sopor lenit? Icl denke ut non ante, wie noch nie zuvor. Dis. v. 739: dolor attrahit orbes, besser getrennt at trahit. Auf die lesarten levet für luat 11.570 und diffuderis für diffunderis VI.56, die durch conjectur aus dem texte versclıwunden sind, lıat mich eigene conjectur zurückgefülırt; sie müssen also doch auch etwas für sich haben.

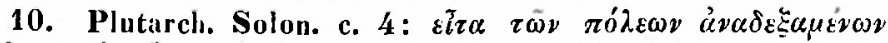

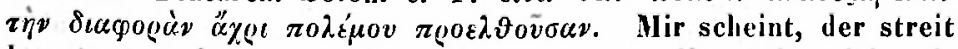
konnte erst dann $2 u m$ kriege fülıren, wenn die städte sich seiner angenommen hatten; das verlangt aber $\pi \rho \circ \varepsilon \lambda$ fovow̃v, so dass das eine participium dem andern subordinirt ist; vgl. Meier pliilol. blatter I, s. 12, Boissonade ad Aristaenet. p. 256, Pflugk ad Eurip. Hecub. 90, Walz ad Arsen. p. 53 u.s.w. Das. c. 15 ist der erste vers des solonischen bruclistücks so zu ergänzen :

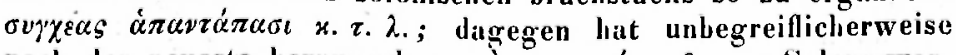

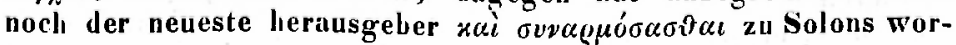
ten gezogen, wogegen sclion das gesetz des irochäischen verses

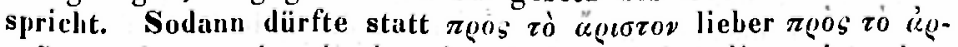
$\chi(c i o y$ zu lesen sein, da der sinn doch zunächst dieser ist: dass 
eine radicale reform zu grosse wirren veranlasse, die sicli scliwer wieder in die alte ordnung bringen lassen. Dass diese reform unter einem idealischen gesiclitspuncte unternommen werden könne, den abor Solon aus eben diesem grunde uicbt bibe verfolgen

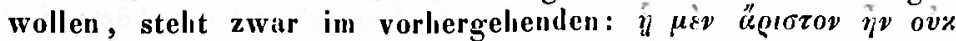
$\dot{\varepsilon} \pi \hat{\gamma} \alpha \gamma \varepsilon \nu$ i $\tau_{\rho \varepsilon i \alpha \nu}$ x. $\tau . \lambda$., brauclit aber in dem vorliegenden ge-

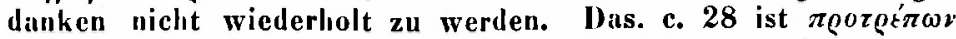
auffälig, da das folgende doclı eigentlich keine aufmunterung enthält; iclı dïclıte vielmehr $\pi \varrho 0 \varsigma \varepsilon \varepsilon \omega \nu$. V. Phoc. c. 2: $\tilde{\omega} \xi \pi \varepsilon \varrho$

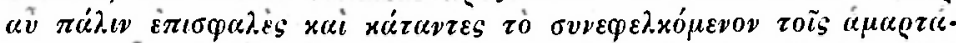

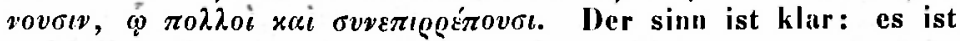
gefälırliclı sich von den felılern seiner mitbürger aus sclıwäche mit fortreissen zu lassen; aber was soll der matte zusatz: „, wozu sich viele auch zugleich hinneigen," zumal das xai, worin doch gar keine steigerung gegen dis vorhergehende liegen kann? Es ist külın; aber ich zögere doch nicht vorzuschlagen: $\tau$ io $\sigma v v \varepsilon q \varepsilon \lambda$ -

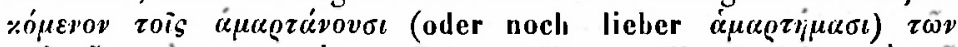

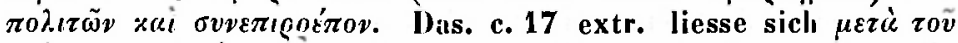

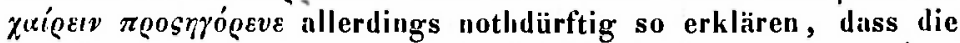
anrede, der name zu anfang der briefe mit dem zusatze $\chi \alpha{ }^{\prime} \chi^{\prime}{ }^{\prime} y$ verbunden worden sei; aber einfacher und sinngemässer scheint mir doch $\mu \varepsilon \tau \dot{c}$ zoṽzo. V. Brut. c. 40 deutet schon die lesart der meisten handsebriften $\dot{\varepsilon} \pi \alpha \iota \tilde{\omega} \nu z o$ darauf, dass hinter $\dot{\varepsilon} \pi \alpha u \tilde{\omega} \nu$ etwas herausgefallen ist, was den folgenden satz anknüpft; iclı

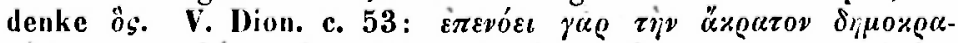

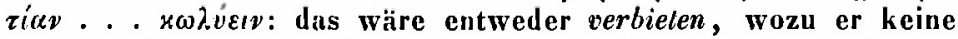
macht hatte, oder verhindern, wähırend sie schon bestand; der spracligebrauch verlangt vielmelir $x \alpha \tau \alpha \lambda v \varepsilon(v$. Das. c. $57: \pi \alpha i-$

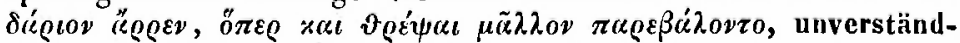
lich; vielleiclit $\mu \alpha \sigma \tau o \nu$ für $\mu \tilde{u} \lambda \lambda_{o \nu}$, obgleicli aucl so die construction lückenhaft bleibt.

11. Arrian. diss. Epietet. I. 2. 4 schreibt Schweighäuser

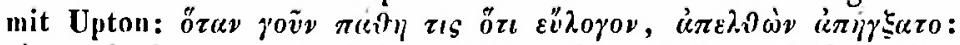
aber wie kann $\pi \alpha \vartheta \varepsilon i \nu$ heissen leni quadam opinione affici? Frülier

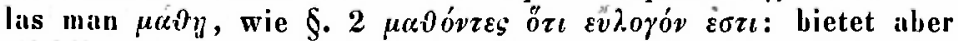
wirklich eine gute handschrift $\pi \dot{\varepsilon} \vartheta \emptyset$, so möchte dieses lieher die

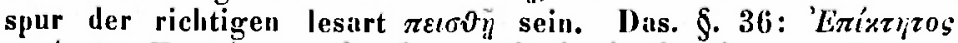

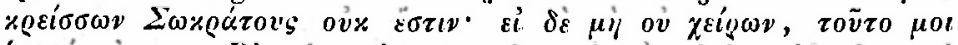
ixevov coziv. Die doppelte negation $\mu \eta$ ov wird wolıl niemand melır mit Hieronymus Wolf für eine verstärkung halten; aber auch eine trennung derselben durch interpunction gibt keinen

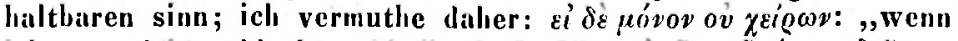

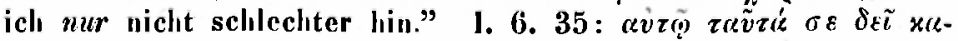
$\tau \varkappa \sigma \varkappa \varepsilon v a ́ s \varepsilon \iota \nu$, olıne alle reclitfertigung der zweiten person, da vorlier und nachher nur von Herikles die rede ist und $\varepsilon i^{\prime}$ rijp

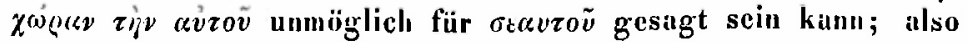




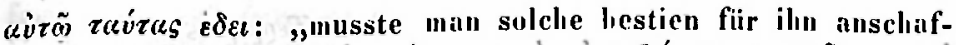

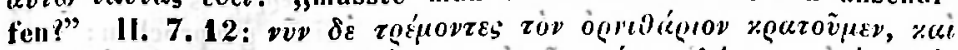

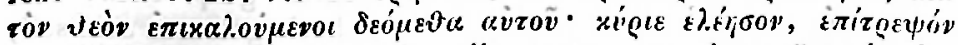

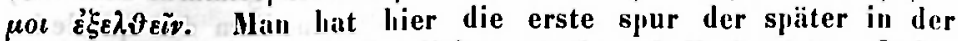
clıristlichen kirche gebräuchlichen bittformel hyrie eleison finden wollen; ich halte jedoch dieses zusimmentrefin'n fiir ganz zufiil-

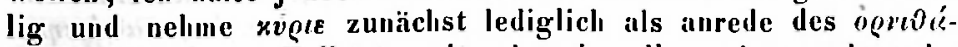
gios, wie sie zu Epiktets zeit schon im allgemeinen gebriuche vorkommt, vgl. 1. 29. 48 ; II. 15. $15 ; 16$. 13 ; 20. 30 ; III. 10. $15 ; 22.38 ; 23$. 11; wälırend die beziehung anf die gottheit vielmelır in dem $\dot{\lambda} \varepsilon \eta \eta_{\sigma o v}$ zu liegen scheint, nur diss dann allerdings besser xai $\omega s, \vartheta \varepsilon \dot{\nu}$ gelesen würde: „, wir klammern uns an den vogelschauer und richten bitten an diesen, wie sie uur der gottlieit zukommen." Wo möglich noch weniger übrigens mag sich die clıristliche kirchengesehichte der stelle IV. 7. 6 bedicnen, wo sicher nur der unverstand eines klösterlichen abschiei-

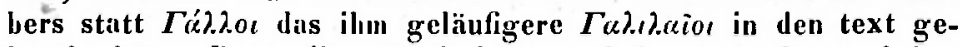
brachit hat. Dass die ascetischen erscheinungen des urchristlichen lebens auch von einem Griechen nicht aus blosser gewolın-

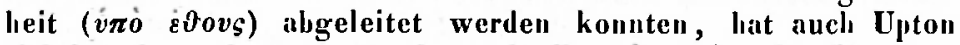

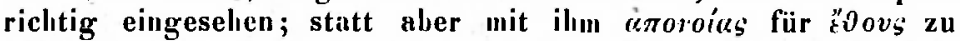
schreiben, ist doch uuser ausweg der mildere, durch den wir statt der cliristen, von welchen es noch nicht einmal sicher ist, ob sie so frülı schon Galiläer genannt worden, die bettelpfatlen der Kybcle erlialten.

12. Unter den wilden thieren, welche in mythischer zeit Griechenland verheerten, zälılt Pausan. 1. 27. 9 auch einen parnasischen löwen auf, von welchem die sonstige griechische mythologie nichts weiss. Drei löwen, sagt scliol. Theocr. X11. 6, habe Herakles erlegt, den nemeischen, den lelikonischen (vgl. Ptolem. Hephaest. 11, p. 15 Roulez), und den lesbischen oder (nach Ungers conjectur Theb. parad. p. 401) thespischen - sollen wir diesen den parnasisclien als vierten linzufügen? lch

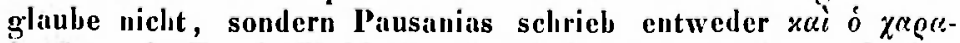
Squĩos, wie Zenob. VI.39 den thespischen nennt, oder noch eher

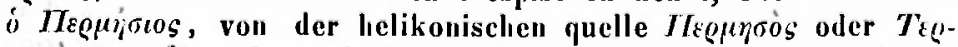
myos, wofür wir auch bei Hesiod. theog. v. 5 in einer handsclirift Ilugnjoos substituirt finden. Dieser wäre also dem helikonisclıen identiseh; und wenn wir kaum zweifelı dürfen, dass es derselbe ist, von dem Statius Theb. 1. 485 sagt:

\section{quem per Teumesia tempe}

Amphitryoniudes frachum iuvenilibus armis

anle Cleonaei vestitur proelia monsiri,

so wird auch dieser teumessische mit dem helikonischen rins scin, maı möge nun dessen verlälthiss zu dem thespischen und kithärouischen (Apollod.11. 4.9) auflassen wie man wolle. Ceber 


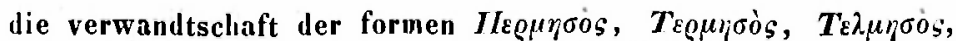

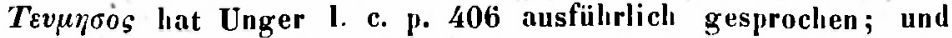
wenn das eine ein berg, das andere ein bach gewesen sein soll, so stehn ja auch diese begriffe durch das medium der quelle im innigsten zusammenhange. Das. c. 28. 9 ist die lesart jedenfalls

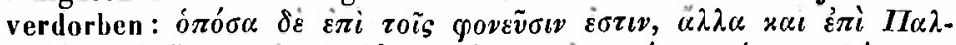

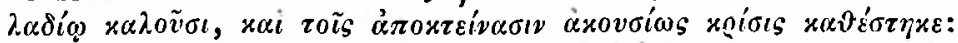

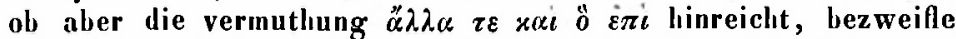
ich, da auch die andern nachher einzeln aufgezähıt sind, was sonst gerade durch die wendung $\alpha \lambda \lambda_{0} \quad \tau \varepsilon$ x $\alpha i$ erspart zu werden pflegt; vielleiclit steckt in $\alpha 2 \lambda \lambda a$ das zahlzeichen $\alpha$ mit folgendem $\mu \varepsilon \dot{\nu}$ - denn $\mu$ und $\lambda \lambda$ werden oft verwechselt - und wäre dann

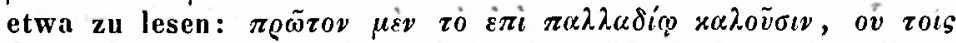

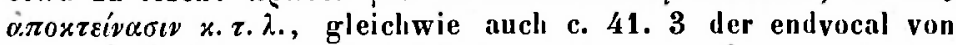

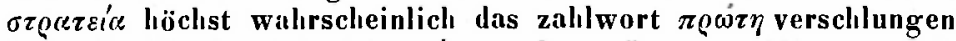
hatt. C. 29.4 würde ich $\mu v \dot{\eta} \mu \alpha z \alpha$ für $\mu \nu \tilde{\eta} \mu \alpha$ vorschlagen; noch

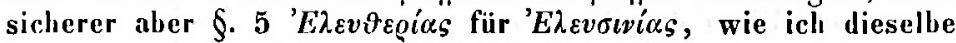
änderung auch bei Nonnus XXVII. 307 in Ritschl's rhein. mus.

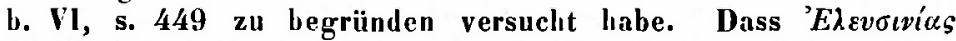
falsch ist, hat schon Böcklı im Berliner lectionskataloge 1822 bemerkt und aus Strabo IX, p. 405 'E $\lambda$ ewvias vorgeschlagen; aber dieses Eleon scheint selbst ein theil des tanagräischen gebiets gewesen zu sein und konnte folglich keine gränze $\pi 00 s$ Tavoyoxiovs haben, wälırend Eleutherä bekanntlich frülızeitig init Attika verbunden den geeigneten platz zu einem gränzgefechte zwischen Athenern und Böotern darbot. C. 34.3 ist die con-

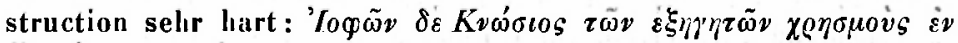

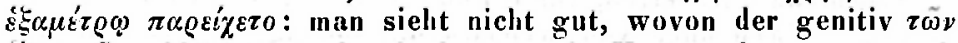
$\varepsilon \xi_{r \gamma \gamma \tau \tilde{\omega} \nu}$ ablängt; und wie kommt ein Kreter als exeget nach

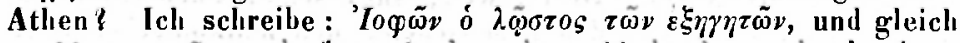

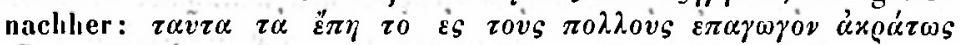

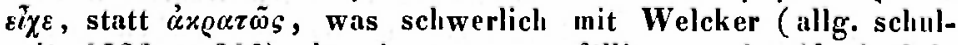
zeit. 1832, s. 210) als , ,der menge gefällig ausscbweifeud, d. h. sehr demokratischen geistes" zu erklären sein dïrfte. C. 35.2 scheint statt 'A $\sigma \times \eta \tau \tau^{\prime} \delta \delta v$ besser 'A $A \times \lambda \eta \pi u$ ádov gelesen zu werden;

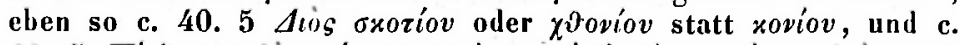

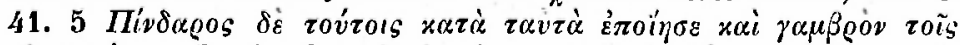

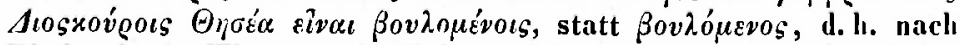
Pindar hatte Theseus die Helena nicht geraubt, sondern mit einwilligung ilırer brüder geebliclit, bis er sie verliess, um Peirithoos auf seinem zuge in den Hades zu begleiten. Endlich bezweifle ich, dass das epitheton Kagwos, das dem pyranidenähnlichen Apoll c. 44.3 beigelegt wird, überhaupt sprachlich zu rechtfertigen sei; seine rohe kunstform, wie die ursprïngliche bevölkerung von Megaris selbst dïrften unbedenklich Kagaxos empfehlen, wofür auch nicht einmal nach der aualogie von $K a$ (aiv) mit verändertem accent Kágıvos ausreiclite; denn jenes ist 
femininum von Kúg wie howîng ron ñons, woraus niemandem einfallen wird ein masculinum ygáiros diir ỵäixòs zu bilden.

Göttingen.

K. F. Hermann.

\section{Zwei fragmente bei Athenäus.}

Die zwei letzten verse des wichtigen bruchstüclis aus der kyklischeu 'Thebais bei Atben. XI. p. M65 bat G. Hermann zwiefacl emendirt. Oedipus flucbt seinen sölıen:

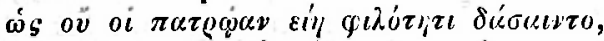

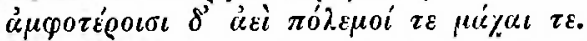

Hierfür schrieb Hermann zum Oedipus Kol. 1377:

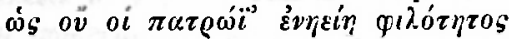

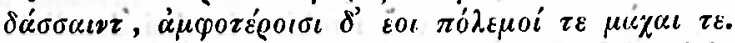

Diese sinnreiche verbesserung nahm W. Dindorf im Athenäus in den text auf, auch befolgten sie Wunder Oed. Col. p. 19 und Diibner in den fragmenten des epischen cyclus hinter dem didotschen Homer. Dagegen erhob einwendungen wider sie v. Leutscb Theb. cycl. rel. p. 38 und in einem nacbtrag zu dieser sclirift in der allgem. schulzeitung; und läugnen kann man nicht, dass

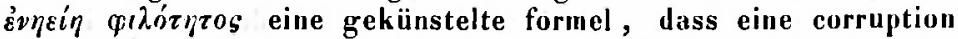

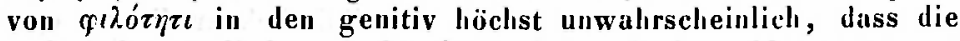
aufgehobene gliederung des fluchis in zwei abgeschlossene verse nicht vortheilhaft und dass das durch $\vec{\varepsilon} 0 \iota$ verdrängte àz fast unentbehrlich ist. Aus diesen oder andern grïnden hat dilier Hermann selbst die emendation aufgegeben und de Aeschyli Trilogiis 'Theb. p. 8 dafür geschrieben :

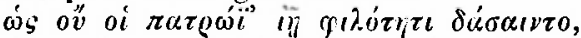

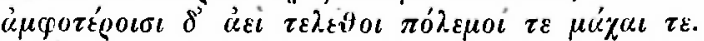

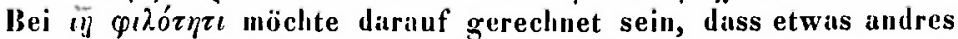
aus den verdorbenen vier huchstaben nicht herauszubringen sei. Aber gerade das weggeworfene $N$ in $\pi \alpha \tau \varrho \varphi \alpha \nu$ ist wichtig, da in den simmlosen stellen der verse bei Athenäus so viel urkundliches hängen geblieben ist. Auch im letzten vers möchte eher

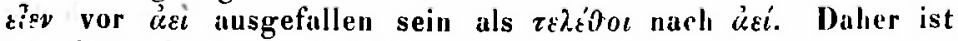
mir folgendes wallirscheinlicher:

$$
\begin{aligned}
& \omega \mathrm{s} \text { ö }
\end{aligned}
$$

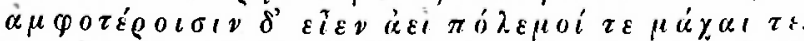

Die véu stimmen mit den hesiodischen schanafen des Oedijus überein, um welche die söhne stritten. II. XI, 480 \&े rínti $\sigma \varkappa \iota \varepsilon \rho \tilde{a}, \nu \varepsilon \mu \varepsilon \varepsilon \sigma \sigma c$ wiederholt bei Nikander.

Aus der Alkmäonis fülırt Atlıenäus XI. p. $400 \mathrm{~b}$ wegen der

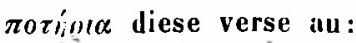

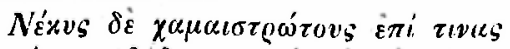

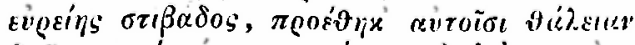

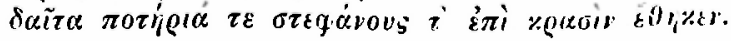

